\title{
MATHEMATICAL MODELLING OF THE DYNAMICS OF COVID-19 PANDEMIC
}

\author{
J.N. NDAM* \\ Department of Mathematics, University of Jos, Nigeria \\ ${ }^{*}$ Corresponding author: ndamj@unijos.edu.ng
}

Received Oct. 22, 2020

\begin{abstract}
A mathematical model to describe the dynamics of COVID-19 was formulated and analysed. The model is aimed at addressing the peculiarities of the dynamics of COVID-19 as identified by researchers as much as possible. Standard analysis indicates the existence of a disease-free equilibrium for the model which is locally-asymptotically stable when the basic reproduction number is less than unity. Conditions for the existence and stability of the endemic equilibria were determined. A backward bifurcation was found to be possible and triggered by the clinical progression of symptoms from asymptomatic to mild and to severe symptoms. Numerical simulation shows no significant difference in the dynamics of the asymptomatic and those with mild symptoms. The result also also shows that strict enforcement of quarantine can help contain the disease.
\end{abstract}

2010 Mathematics Subject Classification. 92B05; 92D30.

Key words and phrases. COVID-19; asymptomatic; basic reproduction number; bifurcation; endemic equilibrium; clinical progression of symptoms.

\section{InTRODUCTION}

In December 2019, an outbreak of a new disease was reported in Wuhan city, Hubei Province of China. The disease is caused by a Coronavirus, hence it was named Coronavirus Disease 2019 (COVID-19), by the World Health Organisation (WHO) [1]. The disease had spread to nearly the whole world by February 2020, leading to its declaration by WHO as a global pandemic on March 11, 2020 ( [2], [3]). One of the unique features of the disease that makes it dangerous is the rate at which it spreads in a population. By March 25, 2020, close to 500,000 cases of the disease had been reported worldwide [4]. Wang, et al [5] gave a clinical

DOI: 10.28924/APJM/8-12 
classification of the cases as mild, ordinary and severe. One of the major challenges to curbing the transmission of COVID-19 is the asymptomatic infections ( [5], [6]), as infected people who show little or no symptoms are least suspected and can easily be left out of any form of isolation or quarantine. Li, et al [7] reported that about 79\% of documented cases are as a result of unreported infections. Furthermore, Sun et al [8] also expressed concerns that COVID-19 presents with asymptomatic infections capable of perpetuating the transmission of the disease. Based on laboratory tests, He, et al [9] inferred that the peak at which the infected person is most infectious is on or before the onset of symptoms of COVID-19. Another serious challenge to the control of COVID-19 is the continuous importation of cases into the population from infected areas [10], hence the introduction of lockdowns, and suspension of both local and international flights in many countries and territories worldwide. The impacts of some of the control measures such as lockdown and isolation have been investigated in [11]. A good number of research work has also been carried out on the Mathematical modelling of COVID-19. Though the dynamics of COVID-19 is similar to other diseases such as SARS and MERS, there are a few peculiarities that need to be considered in any mathematical model of the disease. Some of the mathematical models have considered population compartments such as the susceptibles $S(t)$, the exposed $E(t)$, Presymptom $P(t)$, the symptomatic infectious $I(t)$ and the asymptomatic $A(t)$ [?]. Kochanczyk, et al [13] considered an SEIR model of COVID-19 in which the exposed compartment was subdivided into a cascade of multiple exposed states to take into account the fact that the latent period follows the Erlang distribution. Frost, etal [14] used an SECIR model, similar to the work of Mandal, et al [?], in which the infected class was divided into the asymptomatic, the symptomatic and the severe cases, to predict the dynamics of COVID-19 in West African countries under various lockdown regimes. Other mathematical and statistical models of interest on COVID-19 includes those of Chen, et al [16], Ndairou, et al [17], Zhang, et al [18] and Chakraborty, et al [19]. There are reports that children from 0-14 years are less susceptible to COVID-19 infection than adults aged 15-64 years [20]. The implication of this finding is that there is need to consider age-dependent mathematical models of COVID-19. However, we shall not consider the age-dependent model in this work, but it is a topic for future research. We shall subdivide the infected compartment into three, the asymptomatic, those with mild symptoms and those with severe symptoms as suggested by some of the research findings cited above. Quarantine of the exposed individuals is also a critical method for containing the spread of COVID-19 [2], as confirmed by Ngonghala, 
et al [21] who investigated the effects of interventions such as quarantine, isolation, socialdistancing, and the use of face masks in public in the eradication of COVID-19. It is expected that those who are known or even suspected to have been exposed to the disease should immediately be quarantined or isolated and tested. Ngonghala, et al [21] considered the case of asymptomatic transmission to include those with mild symptoms. However, in the current research, the asymptomatic and those with mild symptoms are classified into separate population compartments. Roda, et al [22] on the other hand compared the SIR and SEIR models of COVID-19 and concluded that the SIR model represented the dynamics of COVID19 better than the SEIR model. We shall take into account key population compartments such as the susceptible, the exposed, the quarantined, the infected and the recovered, as well as dividing the infected compartment into three, so as to capture the peculiarities of COVID-19 dynamics. Subsequent sections of this paper are organised as follows: Section 2 will be dedicated to the mathematical formulation of the model, local stability analysis of equiliibria is the subject of section 3 , while simulations and discussion will be considered in section 4 , and the conclusion will be in section 5 .

\section{Mathematical Formulation of the Model}

A mathematical model for the dynamics of Covid-19 is formulated and analysed, based on the general disposition of medical experts on the transmission dynamics of the disease. We divide the population into the following compartments: the susceptibles $S(t)$, the exposed $E(t)$, the quarantined $Q(t)$, the infected $I(t)$ and the recovered $R(t)$. Literature suggests that the infected class can be subdivided into the asymptomatic or presymptomatic, those with mild symptoms and those with severe symptoms. The subdivision of the infected class is necessary as it has implications for the management of COVID-19 transmission. In particular, it helps medical personnel to accurately prioritise the treatment of patients.Thus, we let $I(t)=I_{1}(t)+I_{2}(t)+I_{3}(t)$, where $I_{1}(t)$ represents the asymptomatic, $I_{2}(t)$ are those with mild symptoms, while $I_{3}(t)$ are those with severe symptoms. 


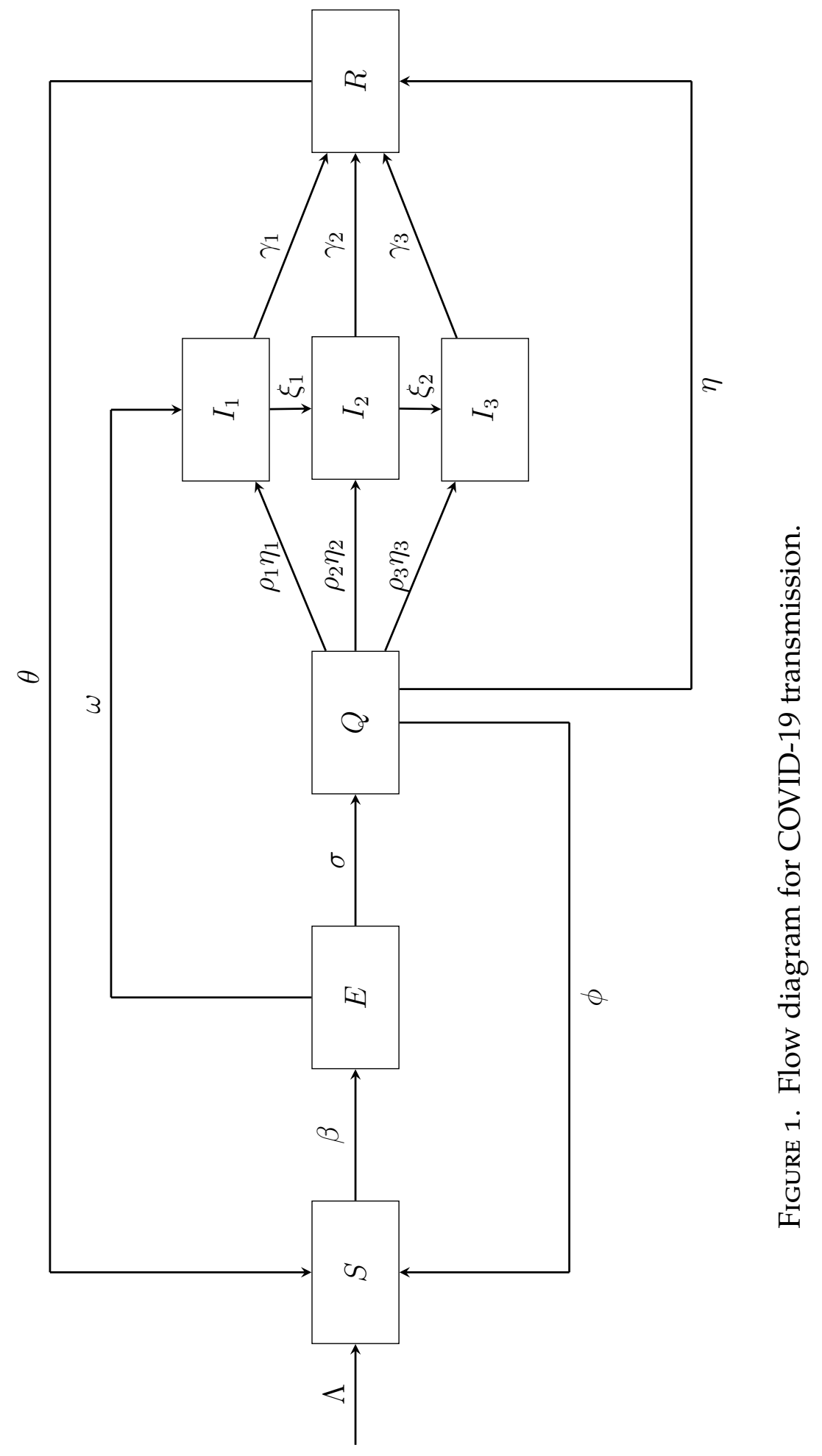


The flow diagram of the transmission dynamics of the model based on these compartments is shown in Fig. 1. From the flow diagram, some exposed persons may not be quarantined, and thus move directly into the infected compartment at the rate $\omega E$, and those quarantined, tested and found negative move back into the susceptible population at the rate $\phi Q$. Similarly, recovered persons who eventually loose immunity become susceptible again at the rate $\theta R$. Here, $\omega=\sum_{j=1}^{n} \omega_{j}$, representing the proportions of the exposed that go into the asymptomatic, those with mild and severe symptoms respectively. The governing equations for the model then become

$$
\begin{aligned}
& \frac{d S}{d t}=\Lambda+\phi Q+\theta R-S \sum_{j=1}^{n} \beta_{j} I_{j}-\alpha S \\
& \frac{d E}{d t}=S \sum_{j=1}^{n} \beta_{j} I_{j}-\left(\alpha+\sigma+\sum_{j=1}^{n} \omega_{j}\right) E \\
& \frac{d Q}{d t}=\sigma E-\left(\alpha+\phi+\sum_{j=1}^{n} \eta_{j}\right) Q \\
& \frac{d I_{j}}{d t}=Q \sum_{j=1}^{n} \rho_{j} \eta_{j}+E \sum_{j=1}^{n} \omega_{j}-\sum_{j=1}^{n}\left(\alpha+\delta_{j}+\gamma_{j}\right) I_{j}-\sum_{j=1}^{n-1} \xi_{j} I_{j}+\sum_{j=1}^{n} \xi_{j-1} I_{j-1} \\
& \frac{d R}{d t}=\sum_{j=1}^{n} \gamma_{j} I_{j}+Q \sum_{j=1}^{n}\left(1-\rho_{j}\right) \eta_{j}-(\alpha+\theta) R
\end{aligned}
$$

with the initial conditions

$$
S(0)>0, E(0)>0, Q(0)>0, I_{j}(0)>0, R(0) \geq 0 .
$$

The parameter $\Lambda$, is the recruitment rate of susceptible individuals, $\sigma$ is the quarantine effort, while $\omega_{j}$ are the rates of progression from the exposed class directly into the infected classes, $\theta$ is the rate at which the recovered lose immunity and become susceptible again, $\phi$ is the proportion of quarantined individuals who tested negative and thus return to the susceptible class and $\delta$ is the disease induced death rate, while $\gamma_{j}$ are the rates of progression from the infected class into the recovered class, $\delta_{j}$ are the disease induced death rates, $\xi_{j}$ are the rates of progression from asymptomatic to mild symptoms and then to severe symptoms, and $0<\rho_{j}<1$ are the proportions of those who escape from quarantine into the infected class at the rates $\eta_{j}$ and $\eta=\sum_{j=1}^{n}\left(1-\rho_{j}\right) \eta_{j}$. The natural death rate, $\alpha$, in all the compartments is not indicated on the flow diagram. 
The feasible region of the model is defined by

$$
\Psi_{n}=\left\{\left(S, E, Q, I_{j}, R\right): S+E+Q+\sum_{j=1}^{n} I_{j}+R \leq \frac{\Lambda}{\alpha}, n=3\right\},
$$

which is positively invariant. The disease-free equilibrium (DFE) is obtained as

$$
E_{0}\left(S, E, Q, I_{j}, R\right)=\left(\frac{\Lambda}{\alpha}, 0,0,0,0\right) .
$$

The basic reproduction number $\mathscr{R}_{j}^{0}$, is obtained by use of the next generation matrix procedure of [23] as follows: Express the equations for the infected compartments as

$$
\frac{d X_{i}}{d t}=F_{i}(X)-V_{i}(X)
$$

where $F_{i}$ represents the new infections in compartment $i$ and $V_{i}$ are the rates of transfer of infections in and out of compartment $i$. Hence from (1), we obtain

$$
\begin{gathered}
F_{1}=S \sum_{j=1}^{n} \beta_{j} I_{j}, F_{2}=F_{3}=0 \\
V_{1}=\left(\alpha+\sigma+\sum_{j=1}^{n} \omega_{j}\right) E, V_{2}=\left(\alpha+\phi+\sum_{j=1}^{n} \eta_{j}\right) Q-\sigma E
\end{gathered}
$$

and

$$
V_{3}=\sum_{j=1}^{n}\left(\alpha+\delta_{j}+\gamma_{j}\right) I_{j}+\sum_{j=1}^{n-1} \xi_{j} I_{j}-\sum_{j=1}^{n} \xi_{j-1} I_{j-1}-E \sum_{j=1}^{n} \omega_{j}-Q \sum_{j=1}^{n} \rho_{j} \eta_{j} .
$$

Hence the Jacobian matrices for $F$ and $V$ evaluated at the disease-free equilibrium, $E_{0}$, are

$$
F=\left(\begin{array}{ccc}
0 & 0 & \frac{\Lambda}{\alpha} \sum_{j=1}^{n} \beta_{j} \\
0 & 0 & 0 \\
0 & 0 & 0
\end{array}\right)
$$

and

$$
V=\left(\begin{array}{ccc}
\alpha+\sigma+\sum_{j=1}^{n} \omega_{j} & 0 & 0 \\
-\sigma & \alpha+\phi+\sum_{j=1}^{n} \eta_{j} & 0 \\
-\sum_{j=1}^{n} \omega_{j} & -\sum_{j=1}^{n} \rho_{j} \eta_{j} & \sum_{j=1}^{n}\left(\alpha+\delta_{j}+\gamma_{j}\right)+\sum_{j=1}^{n-1} \xi_{j}
\end{array}\right)
$$

where $\xi_{0}=0$, as there is no such progression into $I_{1}$ with that rate. The inverse of the matrix $V$ is obtained as

$$
V^{-1}=\left(\begin{array}{ccc}
\frac{1}{\alpha+\sigma+\sum_{j=1}^{n} \omega_{j}} & 0 & 0 \\
\frac{\sigma}{\left(\alpha+\sigma+\sum_{j=1}^{n} \omega_{j}\right)\left(\alpha+\phi+\sum_{j=1}^{n} \eta_{j}\right)} & \frac{1}{\alpha+\phi+\sum_{j=1}^{n} \eta_{j}} & 0 \\
\frac{\sigma \sum_{j=1}^{n} \rho_{j} \eta_{j}+\left(\alpha+\phi+\sum_{j=1}^{n} \eta_{j}\right) \sum_{j=1}^{n} \omega_{j}}{|V|} & \frac{\left(\alpha+\sigma+\sum_{j=1}^{n} \omega_{j}\right) \sum_{j=1}^{n} \rho_{j} \eta_{j}}{|V|} & \frac{1}{\sum_{j=1}^{n}\left(\alpha+\delta_{j}+\gamma_{j}\right)+\sum_{j=1}^{n-1} \xi_{j}}
\end{array}\right),
$$


where

$$
|V|=\left(\alpha+\sigma+\sum_{j=1}^{n} \omega_{j}\right)\left(\alpha+\phi+\sum_{j=1}^{n} \eta_{j}\right)\left(\sum_{j=1}^{n}\left(\alpha+\delta_{j}+\gamma_{j}\right)+\sum_{j=1}^{n-1} \xi_{j}\right) .
$$

Therefore, $\mathscr{R}_{j}^{0}$ is the spectral radius of the matrix $\left(F V^{-1}\right)$, given by

$$
\mathscr{R}_{j}^{0}=\frac{\Lambda \sum_{j=1}^{n} \beta_{j}\left[\sigma \sum_{j=1}^{n} \rho_{j} \eta_{j}+\left(\alpha+\phi+\sum_{j=1}^{n} \eta_{j}\right) \sum_{j=1}^{n} \omega_{j}\right]}{\alpha\left(\alpha+\sigma+\sum_{j=1}^{n} \omega_{j}\right)\left(\alpha+\phi+\sum_{j=1}^{n} \eta_{j}\right)\left(\sum_{j=1}^{n}\left(\alpha+\delta_{j}+\gamma_{j}\right)+\sum_{j=1}^{n-1} \xi_{j}\right)}
$$

\section{Existence and Local Stability Analysis of Equilibria}

To investigate the local stability of the disease-free equilibrium, we evaluate the Jacobiam matrix of the system (1) at the disease-free equilibrium point $E_{0}$ to obtain

$$
J_{E_{0}}=\left(\begin{array}{ccccc}
-\alpha & 0 & \phi & -\frac{\Lambda}{\alpha} \sum_{j=1}^{n} \beta_{j} & \theta \\
0 & -\left(\alpha+\sigma+\sum_{j=1}^{n} \omega_{j}\right) & 0 & \frac{\Lambda}{\alpha} \sum_{j=1}^{n} \beta_{j} & 0 \\
0 & \sigma & -\left(\alpha+\phi+\sum_{j=1}^{n} \eta_{j}\right) & 0 & 0 \\
0 & \sum_{j=1}^{n} \omega_{j} & \sum_{j=1}^{n} \rho_{j} \eta_{j} & -\sum_{j=1}^{n}\left(\alpha+\delta_{j}+\gamma_{j}\right)-\sum_{j=1}^{n-1} \xi_{j} & 0 \\
0 & 0 & \sum_{j=1}^{n}\left(1-\rho_{j}\right) \eta_{j} & \sum_{j=1}^{n} \gamma_{j} & -(\alpha+\theta)
\end{array}\right)
$$

From (3), $\lambda_{1}=-\alpha$ and $\lambda_{2}=-(\alpha+\theta)$ are eigenvalues. Thus the local stability of the DFE is determined by the eigenvalues of the equations for $E, Q$ and $I_{j}$ whose Jacobian matrix is given by

$$
J_{3}=\left(\begin{array}{ccc}
-\left(\alpha+\sigma+\sum_{j=1}^{n} \omega_{j}\right) & 0 & \frac{\Lambda}{\alpha} \sum_{j=1}^{n} \beta_{j} \\
\sigma & -\left(\alpha+\phi+\sum_{j=1}^{n} \eta_{j}\right) & 0 \\
\sum_{j=1}^{n} \omega_{j} & \sum_{j=1}^{n} \rho_{j} \eta_{j} & -\sum_{j=1}^{n}\left(\alpha+\delta_{j}+\gamma_{j}\right)-\sum_{j=1}^{n-1} \xi_{j}
\end{array}\right)
$$

The matrix in (4) has the characteristic polynomial

$$
\begin{array}{r}
P(\lambda)=\lambda^{3}+\left(A_{j}+C_{j}+D_{j}+\sum_{j=1}^{n-1} \xi_{j}\right) \lambda^{2}+\left[A_{j} C_{j}+\left(A_{j}+C_{j}\right)\left(D_{j}+\sum_{j=1}^{n-1} \xi_{j}\right)\right. \\
\left.-B_{j} \sum_{j=1}^{n} \omega_{j}\right] \lambda \\
+\left(1-\mathscr{R}_{j}^{0}\right)=0
\end{array}
$$

where $A_{j}=\alpha+\sigma+\sum_{j=1}^{n} \omega_{j}, B_{j}=\frac{\Lambda}{\alpha} \sum_{j=1}^{n} \beta_{j}, C_{j}=\alpha+\phi+\sum_{j=1}^{n} \eta_{j}$ and $D_{j}=\sum_{j=1}^{n}\left(\alpha+\delta_{j}+\gamma_{j}\right)$, which are all positive constants. The Routh-Hurwitz criterion guarantees that the roots of the characteristic polynomial (5) all have negative real parts, hence the stability of the DFE, if the 
following conditions hold:

$$
\begin{gathered}
A_{j}+C_{j}+D_{j}+\sum_{j=1}^{n-1} \xi_{j}>0, \\
\left(1-\mathscr{R}_{j}^{0}\right)>0 \Rightarrow \mathscr{R}_{j}^{0}<1, \\
\left(A_{j}+C_{j}+D_{j}+\sum_{j=1}^{n-1} \xi_{j}\right)\left[A_{j} C_{j}+\left(A_{j}+C_{j}\right)\left(D_{j}+\sum_{j=1}^{n-1} \xi_{j}\right)-B_{j} \sum_{j=1}^{n} \omega_{j}\right]>1-\mathscr{R}_{j}^{0} .
\end{gathered}
$$

Hence we have the following result:

Theorem 3.1. The disease-free equilibrium (DFE) of the model (1) is locally-asymptotically stable if $\mathscr{R}_{j}^{0}<1$, and unstable otherwise.

3.1. The Endemic Equilibrium. The endemic equilibrium point of model (1) can be expressed as

$$
\begin{aligned}
S^{*} & =\frac{A_{j} C_{j}\left[\Lambda(\alpha+\theta)+\theta \sum_{j=1}^{n} \gamma_{j} I_{j}^{*}\right]}{A_{j} C_{j}(\alpha+\theta)\left(\alpha+\sum_{j=1}^{n} \beta_{j} I_{j}^{*}\right)-\sigma \sum_{j=1}^{n} \beta_{j} I_{j}^{*}\left[(\alpha+\theta) \phi+\theta \sum_{j=1}^{n}\left(1-\rho_{j}\right) \eta_{j}\right]} \\
E^{*} & =\frac{S^{*}}{A_{j}} \sum_{j=1}^{n} \beta_{j} I_{j}^{*} \\
Q^{*} & =\frac{\sigma S^{*}}{A_{j} C_{j}} \sum_{j=1}^{n} \beta_{j} I_{j}^{*} \\
R^{*} & =\frac{1}{(\alpha+\theta)}\left[\sum_{j=1}^{n} \gamma_{j} I_{j}^{*}+\frac{\sigma S^{*}}{A_{j} C_{j}} \sum_{j=1}^{n} \beta_{j} I_{j}^{*} \sum_{j=1}^{n}\left(1-\rho_{j}\right) \eta_{j}\right]
\end{aligned}
$$

where $S^{*}$ is defined for

$$
A_{j} C_{j}(\alpha+\theta)\left(\alpha+\sum_{j=1}^{n} \beta_{j} I_{j}^{*}\right)>\sigma \sum_{j=1}^{n} \beta_{j} I_{j}^{*}\left[(\alpha+\theta) \phi+\theta \sum_{j=1}^{n}\left(1-\rho_{j}\right) \eta_{j}\right] .
$$

Using the equation for $I_{j}$ in (1) and substituting the values of $S^{*}, E^{*}, Q^{*}$ and $R^{*}$ in (6) yields

$$
\begin{array}{r}
{\left[\Lambda \Psi+\theta \sum_{j=1}^{n} \gamma_{j} I_{j}^{*}\right]\left[\sigma \sum_{j=1}^{n} \rho_{j} \eta_{j}+C_{j} \sum_{j=1}^{n} \omega_{j}\right] \sum_{j=1}^{n} \beta_{j} I_{j}^{*}} \\
-\left\{A_{j} C_{j} \Psi\left(\alpha+\sum_{j=1}^{n} \beta_{j} I_{j}^{*}\right)-\sigma \sum_{j=1}^{n} \beta_{j} I_{j}^{*}\left[\Psi \phi+\theta \sum_{j=1}^{n}\left(1-\rho_{j}\right) \eta_{j}\right]\right\} \\
{\left[\sum_{j=1}^{n} d_{j} I_{j}+\sum_{j=1}^{n-1} \xi_{j} I_{j}-\sum_{j=1}^{n} \xi_{j-1} I_{j-1}\right]=0}
\end{array}
$$


where $d_{j}=\alpha+\delta_{j}+\gamma_{j}$ and $\Psi=\alpha+\theta$. Equation (7) is a family of quadratic equations. To verify this, we pick each member $j=1,2,3$. Hence for $j=1$, equation (7) becomes

$$
a_{11}\left(I_{1}^{*}\right)^{2}+a_{12} I_{1}^{*}=0
$$

where $a_{11}=\left(\Gamma_{1} \mathscr{R}_{1}^{0}-\beta_{1} \Lambda \Psi\right) A_{1} C_{1}+\beta_{1} \sigma \Lambda\left[\phi \Psi+\theta\left(1-\rho_{1}\right) \eta_{1}\right]$ and $a_{12}=\alpha A_{1} C_{1} \Lambda\left(\mathscr{R}_{1}^{0}-1\right) \Psi$.

The solutions of (8) are obtained as $I_{1}^{*}=0$ and

$$
I_{1}^{*}=\frac{\alpha A_{1} C_{1} \Lambda\left(1-\mathscr{R}_{1}^{0}\right) \Psi}{\left(\Gamma_{1} \mathscr{R}_{1}^{0}-\beta_{1} \Lambda \Psi\right) A_{1} C_{1}+\beta_{1} \sigma \Lambda\left[\phi \Psi+\theta\left(1-\rho_{1}\right) \eta_{1}\right]},
$$

where $\Gamma_{1}=\frac{\alpha \theta \gamma_{1}}{\Lambda}$. The root $I_{1}^{*}=0$ corresponds to the disease-free equilibrium point, hence the model has a unique endemic equilibrium, provided $\left(\Gamma_{1} \mathscr{R}_{1}^{0}-\beta_{1} \Lambda \Psi\right) A_{1} C_{1}+\beta_{1} \sigma \Lambda[\phi \Psi+\theta(1-$ $\left.\left.\rho_{1}\right) \eta_{1}\right]$ and $\alpha A_{1} C_{1} \Lambda\left(1-\mathscr{R}_{1}^{0}\right) \Psi$ have the same sign. This implies that a backward bifurcation, which normally affects the threshold value of basic reproduction number cannot occur in this case.

For $j=2$, equation (7) can be expressed as

$$
a_{21}\left(I^{*}\right)^{2}+a_{22} I^{*}+a_{23}=0
$$

where $a_{21}=A_{2} C_{2}\left(\Gamma_{2} \mathscr{R}_{2}^{0}-\beta_{2} \Lambda \Psi\right)+\beta_{2} \sigma \Lambda\left[\phi \Psi+\theta\left(1-\rho_{2}\right) \eta_{2}\right]$,

$a_{22}=\alpha A_{2} C_{2}\left(\mathscr{R}_{2}^{0}-1\right)\left(d_{2}+\xi_{2}\right) \Psi+\beta_{2}\left[A_{2} C_{2} \Psi-\sigma\left(\phi \Psi+\theta\left(1-\rho_{2}\right) \eta_{2}\right)\right] \xi_{1} I_{1}^{*}, a_{23}=\alpha A_{2} C_{2} \Psi \xi_{1} I_{1}^{*}$ and $\Gamma_{2}=\frac{\alpha \theta \gamma_{2}}{\Lambda}$.

Equation (9) has the roots $I_{2}^{*}=\frac{-a_{22} \pm \sqrt{a_{22}^{2}-4 a_{21} a_{23}}}{2 a_{21}}$. Equation (9) has two distinct positive roots if $a_{21}>0$ and $a_{22}<0$, thus guaranteeing the occurrence of a backward bifurcation. The results which follow from the consideration of the properties of the roots of equation (9) are summarised in the following theorem:

Theorem 3.2. For $j=2$, the model equation (1) has

(i) no endemic equilibrium when $a_{21}>0$ and $a_{22}>0$

(ii) a unique endemic equilibrium when $a_{21}<0$ and $a_{22}<0$ or $a_{21}<0$ and $a_{22}>0$.

(iii) two endemic equilibria when $a_{21}>0$ and $a_{22}<0$.

We now establish the stability of the endemic equilibrium using theorem 4 of [?], which asserts that the sign of the parameter $\xi$, defined by

$$
\xi=\frac{1}{2} \sum_{i, j, k=1}^{n} v_{k} w_{i} w_{j} \frac{\partial^{2} f_{k}}{\partial x_{i} \partial x_{j}}
$$


determines the nature of the endemic equilibria close to the bifurcation point, where $v$ and $w$ are the nullvectors of the Jacobian matrix of the system (1) at the DFE. Applying the theorem to the system (1), we obtain all the second derivatives as

$$
\frac{\partial^{2} f_{1}}{\partial I \partial S}=\frac{\partial^{2} f_{1}}{\partial S \partial I}=-\sum_{j}^{n} \beta_{j}, \frac{\partial^{2} f_{2}}{\partial I \partial S}=\frac{\partial^{2} f_{2}}{\partial S \partial I}=\sum_{j}^{n} \beta_{j} .
$$

The remaining second derivatives are all zero. Thus, $\xi=-w_{1} w_{4}\left(v_{1}-v_{2}\right) \sum_{j}^{n} \beta_{j}$, where the eigenvectors $v$ and $w$ can be chosen to be positive. For $v_{1}-v_{2}>0, \xi<0$ and thus the endemic equilibria are locally asymptotically stable near the DFE for $\mathscr{R}_{0}>1$, whereas the DFE equilibrium is locally asymptotically stable for $\mathscr{R}_{0}<1$, as already established. When $v_{1}-v_{2}<0, \xi>0$ and we have a backward bifurcation at $\mathscr{R}_{0}=1$. A backward bifurcation is triggered in this case by the clinical progression of symptoms of COVID-19.

The descriptions of all the baseline parameters used in model (1) as well as their estimated values are given in table 1 . The source of each parameter is also indicated and they will be used in the numerical simulation of the model. 


\begin{tabular}{|c|c|c|}
\hline Parameter & Description & Estimated value \\
\hline$\Lambda$ & Recruitment rate of susceptible individuals & $0.0375[24]$ \\
\hline$\phi$ & Rate of progression from quarantine to the susceptible & $\frac{1}{14}[19]$ \\
\hline$\theta$ & Rate at which the recovered lose immunity & 0.27 estimated [25] \\
\hline$\beta_{1}$ & Rate of infection of the asymptomatic & $0.179[24]$ \\
\hline$\beta_{2}$ & Rate of infection of those with mild symptoms & 0.04 assumed \\
\hline$\beta_{3}$ & Rate of infection of those with severe symptoms & 0.01 assumed \\
\hline$\alpha$ & Natural death rate of all individuals & 0.01 assumed \\
\hline$\sigma$ & Quarantine efforts & $0.4[4]$ \\
\hline$\omega_{1}$ & Rate of progression from the exposed to the asymptomatic & 0.20 assumed \\
\hline$\omega_{2}$ & Rate of progression from the exposed to mild symptoms & 0.30 assumed \\
\hline$\omega_{3}$ & Rate of progression from the exposed to severe symptoms & 0.40 assumed \\
\hline$\rho_{1}$ & Proportion of those who escape from quarantine to asymptomatic & 0.25 assumed \\
\hline$\rho_{2}$ & Proportion of those who escape from quarantine to mild symptoms & 0.20 assumed \\
\hline$\rho_{3}$ & Proportion of those who escape from quarantine to severe symptoms & 0.15 assumed \\
\hline$\eta_{1}$ & Rate of progression from quarantine to the asymptomatic & 0.45 estimated $[27]$ \\
\hline$\eta_{2}$ & Rate of progression from quarantine to mild symptoms & 0.35 estimated [27] \\
\hline$\eta_{3}$ & Rate of progression from quarantine to severe symptoms & 0.20 estimated [27] \\
\hline$\gamma_{1}$ & Rate of recovery of the asymptomatic & 0.12 estimated [25] \\
\hline$\gamma_{2}$ & Rate of recovery of those with mild symptoms & 0.10 estimated [25] \\
\hline$\gamma_{3}$ & Rate of recovery of those with severe symptoms & 0.05 estimated $[25]$ \\
\hline$\delta_{1}$ & Disease induced death rate of the asymptomatic & 0.00 assumed \\
\hline$\delta_{2}$ & Disease induced death rate of those with mild symptoms & 0.012 assumed \\
\hline$\delta_{3}$ & Disease induced death rate of those with severe symptoms & 0.021 estimated [28] \\
\hline$\xi_{1}$ & Rate of progression from asymptomatic to mild symptoms & 0.10 assumed \\
\hline$\xi_{2}$ & Rate of progression from mild to severe symptoms & 0.20 assumed \\
\hline
\end{tabular}

\section{TABle 1. Description of Baseline Parameters for Model (1).}

\section{Simulations and Discussion}

An $S E Q I_{j} R$ model for the transmission of COVID-19, incorporating the key compartments and parameters in the dynamics of the disease is considered. Standard analysis indicates that the DFE exists and conditions for which it is locally asymptotically stable when $\mathscr{R}_{j}^{0}<1$ were determined. The model has two endemic equilibria due to clinical progression of the disease symptoms from asymptomatic to mild symptoms and from mild to severe symptoms. The existence of several endemic equilibria guarantees the possibility of occurrence of a backward 
bifurcation. Consequently, a critical value for the basic reproduction number was obtained using $j=2$. It should be noted that when $j=1$, the model has a unique endemic equilibrium. Several endemic equilibria only exist for $j=2$ and $j=3$ due to the clinical progression of symptoms of the disease. Numerical simulation was also carried out using estimated parameters as well as data from various sources, including [24] and [25] and many others as contained in Table 1. Numerical results show little or no difference between the asymptomatic and those with mild symptoms, hence it is sufficient to subdivide the infected population into two compartments, the asymptomatic and the symptomatic. The three subdivisions could be used only for the purpose of prioritising treatment of patients by medical personnel. The results also indicate that the disease can be contained within 250 days of the outbreak as can be observed in Figure 2, if appropriate measures are put in place as specified by the estimated parameters of the model. Figure 3 illustrates the effect of relaxing quarantine efforts on the control of the pandemic. It can be observed that relaxing quarantine efforts leads to persistence of the disease in the population. Figure 4, on the other hand is the graphical illustration of the impact of clinical progression from asymptomatic to mild and finally to severe symptoms on the dynamics of the disease. It can be observed from Figure 4 that the disease persists when there is no clinical progression from asymptomatic to mild symptoms (that is, $\xi_{1}=0.0$ ). This is consistent with the fact that the disease symptoms of the asymptomatic and those with mild symptoms are not visible to the public. However, clinical progression from mild to severe symptoms has a less impact on transmission of the disease as can be seen in Figure 5, when compared with with Figure 4. Moreover, given that there is no clinical progression of symptoms, the disease will persist in the population as illustrated in Figure 6. 


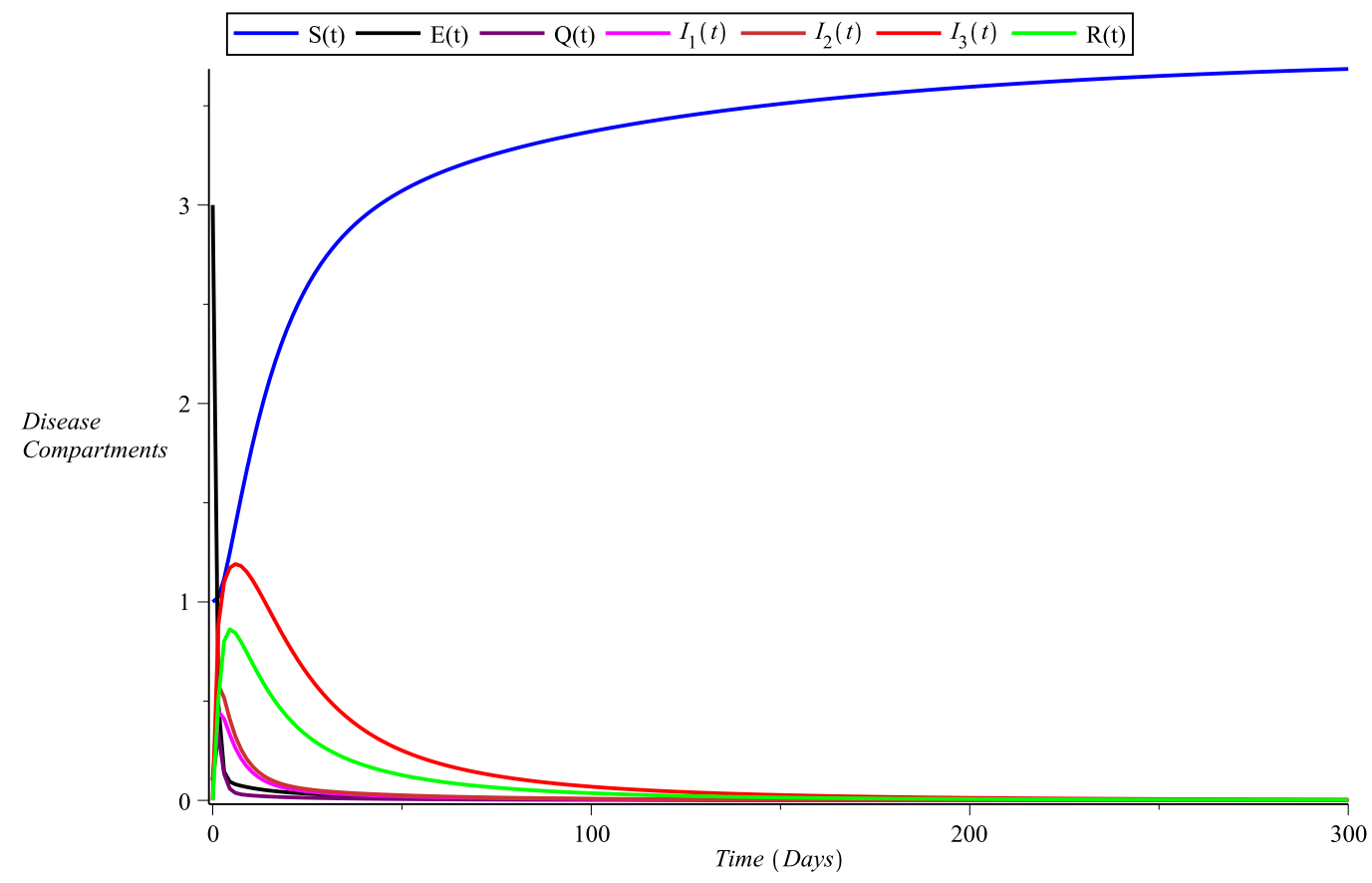

Figure 2. Time course solution for the parameter values contained in Table 1.

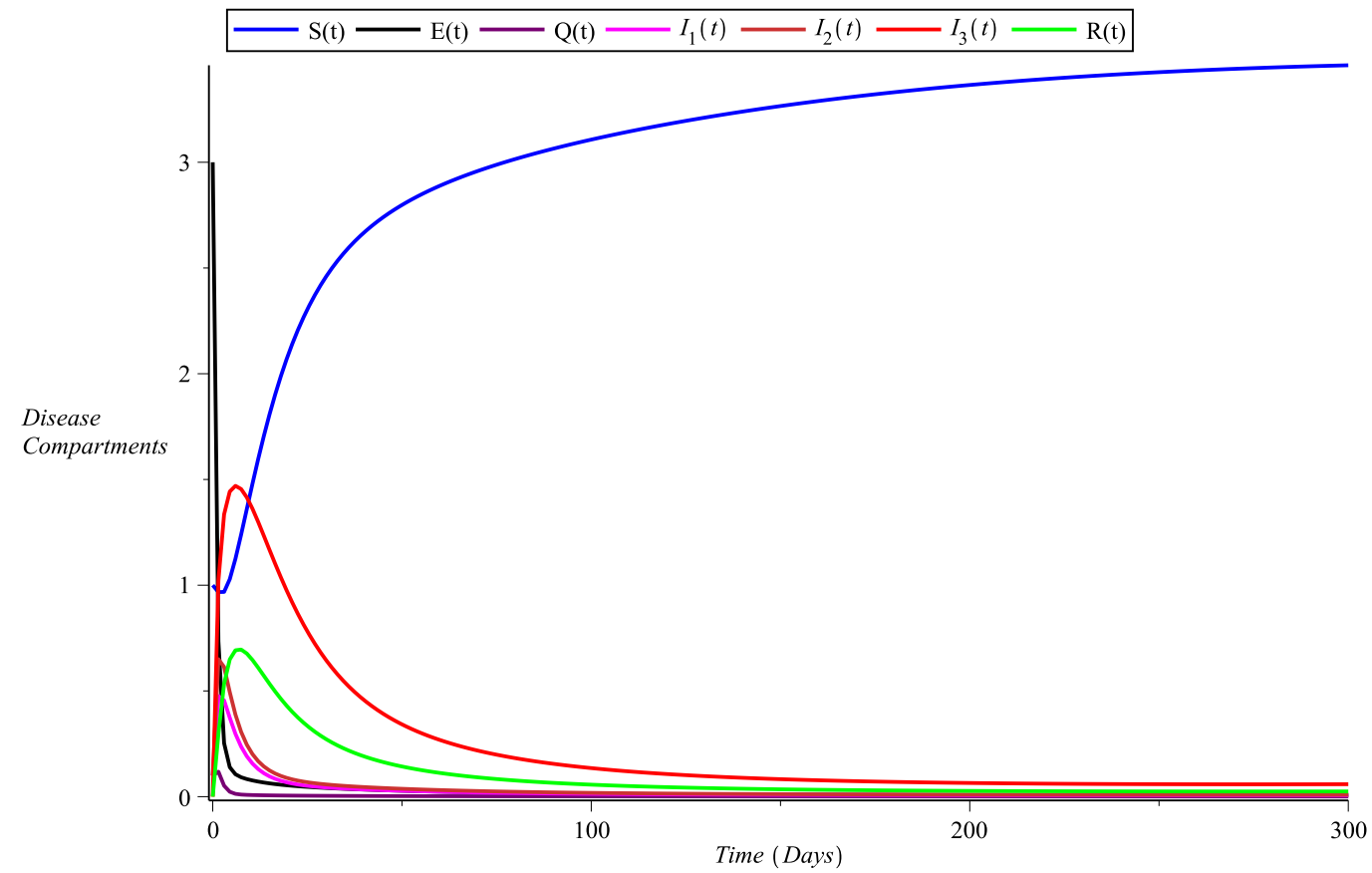

FIGURE 3. Solution with relaxed quarantine from $\sigma=0.4$ to $\sigma=0.1$. 


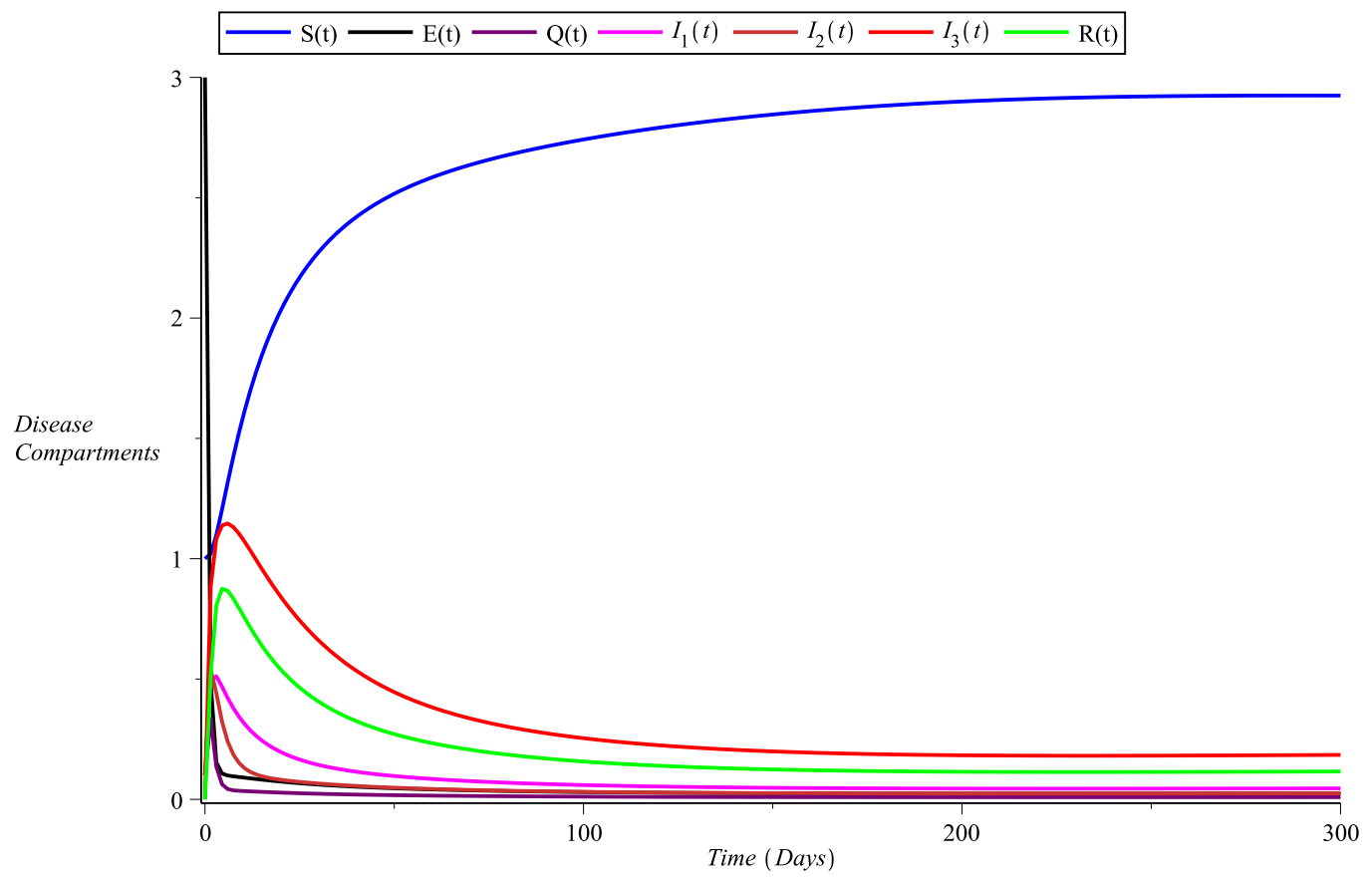

FIGURE 4. Solution with no clinical progression from asymptomatic to mild symptoms $\left(\xi_{1}=0.0\right)$.

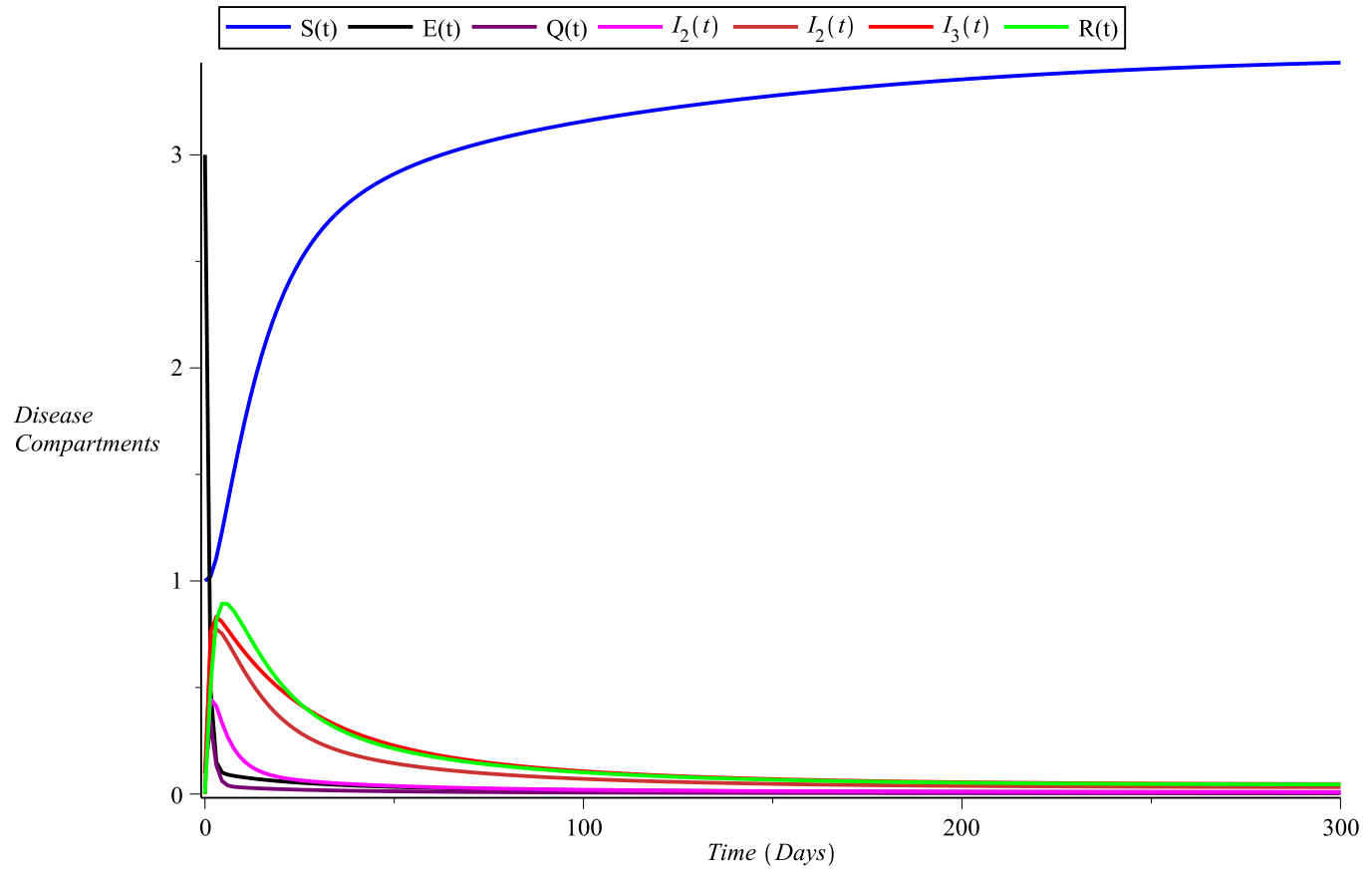

FIGURE 5. Solution with no clinical progression from mild to severe symptoms $\left(\xi_{2}=0.0\right)$. 


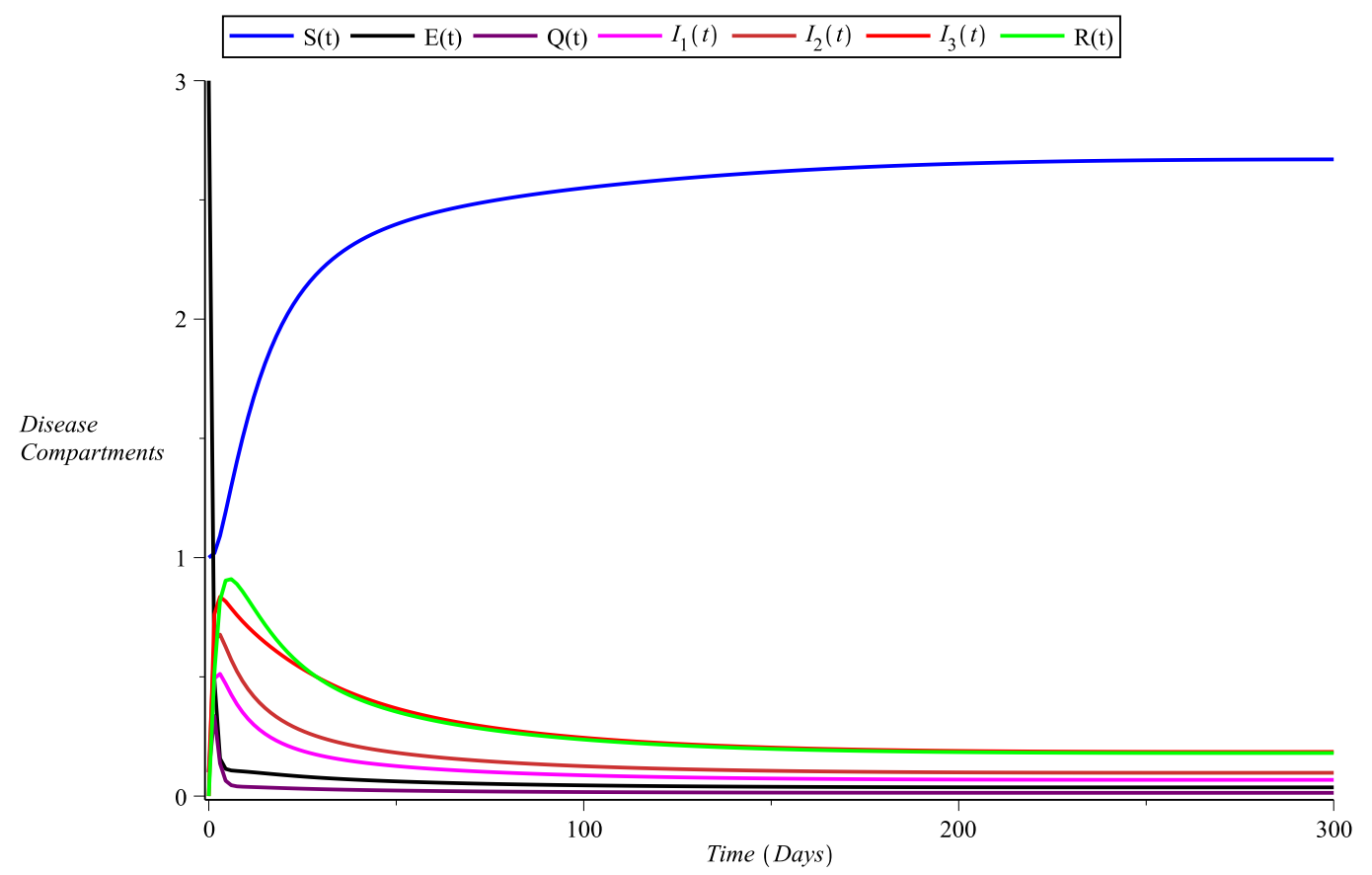

FIGURE 6. Solution with no clinical progression of symptoms $\left(\xi_{1}=0.0, \xi_{2}=0.0\right)$.

\section{Conclusions}

In this paper, we constructed and analysed a model to describe the transmission of COVID-19 based on the population compartments including the susceptibles, the exposed, the quarantined, the infected and the recovered. We established the existence of a disease-free equilibrium which is locally asymptotically stable for $\mathscr{R}_{j}^{0}<1$. We also established conditions for the existence of endemic equilibrium points and the possibility of occurrence of a backward bifurcation due to clinical progression of the disease symptoms. Consequently, a threshold value for $\mathscr{R}_{j}^{c}$ was obtained using the case when $j=2$. Numerical simulation results indicate a little or no difference between the asymptomatic and those with mild symptoms. Consequently, it is sufficient to subdivide the infected class into the asymptomatic and the symptomatic only.

FUNDING

The author declares that there was no funding from any source in support of this research work.

\section{COMPETING INTERest}

The author declares that they have no known competing interest or personal relationship that could have influenced the work reported in this paper. 


\section{ACKNOWLEDGMENTS}

The author acknowledges the assistance of Patricia Azike and James Gbolahan in the construction of the model flow diagram.

\section{REFERENCES}

[1] World Health Organisation, Coronavirus Disease 2019 (COVID-19) Situation Report-22, 11 February, 2020.

[2] World Health Organisation, Coronavirus Disease 2019 (COVID-19) Situation Report-59, 19 March, 2020.

[3] M. Peirlinck, K. Linka, F. Sahli Costabal, E. Kuhl, Outbreak dynamics of COVID-19 in China and the United States, Biomech. Model. Mechanobiol. 19 (2020), 2179 ?2193.

[4] M. Gatto, E. Bertuzzo, L. Mari, S. Miccoli, L. Carraro, R. Casagrandi, A. Rinaldo, Spread and dynamics of the COVID-19 epidemic in Italy: Effects of emergency containment measures, Proc. Natl. Acad. Sci. USA. 117 (2020), 10484?10491.

[5] Y. Wang, Y. Liu, L. Liu, X. Wang, N. Luo, L. Li, Clinical Outcomes in 55 Patients With Severe Acute Respiratory Syndrome Coronavirus 2 Who Were Asymptomatic at Hospital Admission in Shenzhen, China, The J. Infect. Dis. 221 (2020), 1770?1774.

[6] L. Pang, S. Liu, X. Zhang, T. Tian, and Z. Zhao, Transmission dynamics and control strategies of COVID-19 in Wuhan China, J. Biol. Syst. 28 (2020), 543-560.

[7] R. Li, S. Pei, B. Chen, Y. Song, T. Zhang, W. Yang, J. Shaman, Substantial undocumented infection facilitates the rapid dissemination of novel coronavirus (SARS-CoV-2), Science. 368 (2020), 489 ? 493.

[8] J. Sun, W-T. He, L. Wang, A. Lai, X. Ji, X. Zhai, G. Li, M.A. Suchard, J. Tian, J. Zhou, M. Veit and S. Su, COVID-19: Epidemiology, evolution and cross-disciplinary perspectives, Trends Mol. Med. 26 (2020), 483-495.

[9] X. He, E.H.Y. Lau, P. Wu, X. Deng, J. Wang, X. Hao, Y.C. Lau, J.Y. Wong, Y. Guan, X. Tan, X. Mo, Y. Chen, B. Liao, W. Chen, F. Hu, Q. Zhang, M. Zhong, Y. Wu, L. Zhao, F. Zhang, B.J. Cowling, F. Li, G.M. Leung, Temporal dynamics in viral shedding and transmissibility of COVID-19, Nat. Med. 26 (2020) $672 ? 675$.

[10] O.A. Adegboye, A.I. Adekunle and E. Gayawan, Early transmission dynamics of novel coronavirus (COVID19) in Nigeria, Int. J. Environ. Res. Pub. Health,17 (2020), 3054. doi: 10.3390/ijerph17093054

[11] J.N. Ndam, Modelling the impacts of lockdown and isolation on the eradication of COVID-19, Biomath. 9 (2020) 2009107.

[12] K. Liang, Mathematical model of infection kinetics and its analysis for COVID-19, SARS and MERS, Infect. Genet. Evol. 82 (2020), 104306.

[13] M. Kochanczyk, F. Grabowski and T. Lipniacki, Dynamics of COVID-19 Pandemic at Constant and Timedependent Contact Rates, Math. Model. Nat. Phenom. 15 (2020), 28.

[14] I. Frost, G. Osena, J. Craig, S. Hauck, E. Kalanxhi, O. Gatalo, Y. Yang, K. Tseng, E. Schueller, E. Klein and G. Lin, COVID-19 in West Africa: National projections of exposed, contagious, symptomatic \& severe cases, Publication of the Center for Disease Dynamics, Economics and Policy. 2020. 
[15] M. Mandal, S. Jana, S.K. Nandi, A. Khatua, S. Adak and T.K. Kar, A model based on the dynamics of COVID-19: Prediction and control, Chaos Solitons Fractals, 135 (2020), 109889.

[16] T.-M. Chen, J. Rui, Q.-P. Wang, Z.-Y. Zhao, J.-A. Cui, L. Yin, A mathematical model for simulating the phase-based transmissibility of a novel coronavirus, Infect. Dis. Poverty. 9 (2020), 24.

[17] F. Ndairou, I. Area, J.J. Nieto and D.F.M. Torres, Mathematical modelling of COVID-19 transmission dynamics with a case study of Wuhan, Chaos Solitons Fractals, 135 (2020), 109846.

[18] X. Zhang, R. Ma and L. Wang, Predicting turning point, duration and attack rate of COVID-19 outbreaks in major Western countries, Chaos Solitons Fractals, 135 (2020), 109829.

[19] T. Chakraborty and I. Ghosh, Real-time forcasts and risk assessment of novel coronavirus (COVID-19) cases: A data-driven analysis, Chaos Solitons Fractals, 135 (2020), 109850.

[20] J. Zhang, M. Litvinova, Y. Liang, Y. Wang, W. Wang, S. Zhao, Q. Wu, S. Merler, C. Viboud, A. Vespignani, M. Ajelli and H. Yu, Changes in contact patterns shape the dynamics of the COVID-19 outbreak in China, Science, 368 (2020), 1481-1486.

[21] C.N. Ngonghala, E. Iboi, S. Eikenberry, M. Scotch, C.R. MacIntyre, M.H. Bonds and A.B. Gumel, Mathematical assessment of the impact of non-pharmaceutical interventions on curtailing the 2019 novel Coronavirus, Math. Biosci. 325 (2020), 108364.

[22] W.C. Roda, M.B. Varughese, D. Han and M-Y. Li, Why is it difficult to accurately predict tthe COVID-19 epidemic? Infect. Dis. Model. 5 (2020), 271-281.

[23] P. van den Driessche (2017). Reproduction numbers of infectious disease models, Infect. Dis. Model. 2 (2017), 288-303.

[24] United Nations (2019), World Population Prospects 2019, Volume II: Demographic Profiles. Nigerian Centre for Disease Control (NCDC), COVID-19 Situation Report 79, March 18, 2020.

[25] Nigerian Centre for Disease Control (NCDC), COVID-19 Situation Report 79, March 18, 2020.

[26] K. Mizumoto, K. Kagaya, A. Zarebski and G. Chowell, Estimating the asymptomatic proportion of coronavirus disease 2019 (COVID-19) cases on board the Diamond Princess cruise ship, Yokohama, Japan, 2020, Euro Surveill. 25 (10) (2020), 1-5.

[27] World Health Organisation, Coronavirus Disease 2019 (COVID-19) Situation Report-46, 06 March, 2020.

[28] Nigeria Centre for Disease Control (NCDC), COVID-19 Situation Report 173, August 19, 2020. 